\title{
Drainage of a brain abscess secondary to liver abscesses through neuronavigation and aspiration-puncture
}

\author{
Miguel A. Velasco-Castillo, Hugo García-Velasco, Antonio Sosa-Nájera, Carlos A. Tevera-Ovando, and \\ César A. Almendárez-Sánchez* \\ Department of Neurosurgery, “Lic. Adolfo López Mateos” Medical Center, Instituto de Salud del Estado de México, State of Mexico, Mexico
}

\begin{abstract}
Objective: The aim of this study was to evaluate the efficiency of brain abscess drainage by neuronavigation, with a case report and review of the literature. Clinical case: A 45-year-old male with headache, fever, and abdominal pain, presented liver and brain abscesses. The operation was performed by draining the liver abscesses through endoscopy, and brain abscesses by neuronavigation, using a single burr hole and a puncture-aspiration technique on the first attempt, without residual abscesses and a neurological deficit. Conclusion: The drainage of brain abscesses with the described technique is high in safety, precision, and effectiveness, avoiding the performance of a wide craniotomy, with less aggression to healthy tissue.
\end{abstract}

Key words: Brain abscess. Liver abscess. Neuronavigation. Neurosurgery.

\section{Introduction}

Brain abscesses are focal intracranial infections caused by bacteria, micro bacteria, fungi, protozoon, or helminths ${ }^{1,2}$. These can cause a severe inflammatory reaction and neurological deficits in patients with all the repercussions these represents ${ }^{3}$. Therefore, new strategies for their management have been created, such as the development of new antibiotics, more effective culture means, and surgical techniques, emphasizing the minimally invasive. We present the case of a patient with a brain abscess successfully treated through trephine and neuronavigation-guided puncture aspiration technique.

\section{Clinical case}

The patient, a 45-year-old male without any case history of relevance, was admitted with a headache, fever, and abdominal pain at $48 \mathrm{~h}$ of evolution. An abdominal tomography was performed, where we were able to observe multiple hepatic abscesses (Fig. 1). Surgical management was decided, consisting of diagnostic laparoscopic adhesiolysis, hepatic abscess drainage and drain placement, multiple lax and firm adherences from the liver to the epiploon, and abdominal wall and diaphragm, in addition to abscesses in the Couinaud's segments IV and VIII of approximately $1000 \mathrm{cc}$. Subsequently, he remained in post-surgical monitoring with Metronidazole and Ceftriaxone-based antibiotic impregnation for 14 days, with coverage for Entamoeba histoIytica and Streptococcus aureus.

During his internment, the patient scored 14 points on the Glasgow scale (0:4 V:4 M:6), was oriented in space and place but disoriented in time, alert, and awake, speaking fluently and coherently. He repeated and nominated, preserved short, medium, and longterm memory, and conserved calculations and abstractions. He presents isochoric pupils of $3 \mathrm{~mm}$ reactive to

\section{Correspondence:}

*César A. Almendárez-Sánchez

E-mail: cesar2hx@hotmail.com
Available online: $24-12-2020$

Date of reception: 07-06-2020

Date of acceptance: 15-09-2020 DOI: 10.24875/RMU.20000047
Medicina Universitaria. 2020;22(4):172-175 www.medicinauniversitaria.org

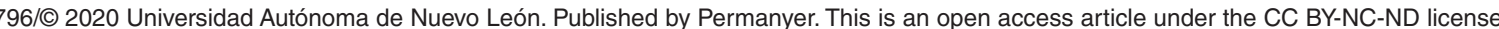
(http://creativecommons.org/licenses/by-nc-nd/4.0/) 




Figure 1. Single-phase abdominal tomography showing multiple well-defined encapsulated liver lesions with heterogeneous density in the sagittal, coronal, and axial sections, corresponding to multiple liver abscesses.

luminous stimulus, without effects to the rest of the cranial nerves, with muscle strength of $5 / 5$ globally using the Daniels scale, preserved sensitivity in all its modalities, preserved muscle stretch reflexes. He did not present data of meningeal irritation, and his vestibulocerebellous system was without alterations.

Subsequently, there were intermittent headaches of a holocranial distribution, with little response despite established medical management. Thus, a simple brain CT scan was performed, where we observed multiple hypodensities located in the left frontal and right temporal region (Fig. 2A). Given said findings, a simple and a MRI scan of the brain were requested, confirming multiple lesions with ring reinforcement when administrating contrast and restrictions marked at diffusion, suggesting a cerebral abscess (Fig. 2B).

We decided on surgical management of the abscess on the right temporal lobe with guided drainage through neuronavigation. First, a tridimensional reconstruction was conducted based on the previously performed MRI, taking as reference the hyperintense ring as the edge of the lesion, calculating its volume with software (Horos v3.3, iOS) reconstructing the pyramidal tract. This information was transferred to the neuronavigator, which was used to create a safe route toward the lesion, avoiding critical neurovascular structures. A linear incision of $3 \mathrm{~cm}$ was performed in the indicated direction until exposing bone tissue with the posterior implementation of the unique trephine of $1 \mathrm{~cm}$, a dural opening in the shape of a cross. The puncture with a plastic catheter in the predetermined trajectory was guided by neuronavigation, avoiding eloquent areas such as the motor, sensitive, visual, and language cortex, as was the subsequent aspiration with a $20 \mathrm{cc}$ syringe, achieving the extraction of purulent material on the first attempt, with posterior irrigation with abundant saline solution in this cavity. Conservative management of the left frontal abscess through antibiotic impregnation was decided on, continuing with ceftriaxone and metronidazole-based double scheme. The material obtained through puncture was sent out for culture, which, $48 \mathrm{~h}$ later, was reported as negative. Primary immunodeficiencies were ruled out during the patient's hospitalization, and an echocardiogram was conducted, which ruled out the presence of valvular structural alteration, which may increase the risk of relapse. After that, the patient was discharged with significant clinical and neurological improvement, with ambulatory antibiotic management for 4 weeks through ciprofloxacin (500 mg orally every $12 \mathrm{~h}$ ) and metronidazole (500 mg orally every $8 \mathrm{~h}$ ). A month after discharge, the patient returned for a review, where he was reported as asymptomatic and neurologically complete. He presents a brain MRI scan with contrast with adequate brain abscess drainage and without data of a relapse (Fig. 3).

\section{Discussion}

There have been reports of metastatic infections in patients with pyogenic hepatic abscesses ${ }^{4}$ with an incidence ranging from $3 \%$ to $12 \%{ }^{5}$, thus making it an infrequent phenomenon. Among its extrahepatic locations, there are the eyes, pleura, meninges, brain, spleen, skin, and soft tissues ${ }^{6}$. There are multiple predisposing factors for its formation, including HIV infection, immunosuppressor medication treatment, natural encephalic barrier disruption resulting from surgical procedures or trauma, mastoiditis, sinusitis, dental infection, or a systemic infection due to endocarditis or bacteremia. Alcoholism and diabetes mellitus have also been identified as predominant risk factors for the formation of these abscesses. Hematogenic implantation has been considered a possible route for the formation of metastatic infections ${ }^{7}$ in up to a third of cases ${ }^{4}$. Other 


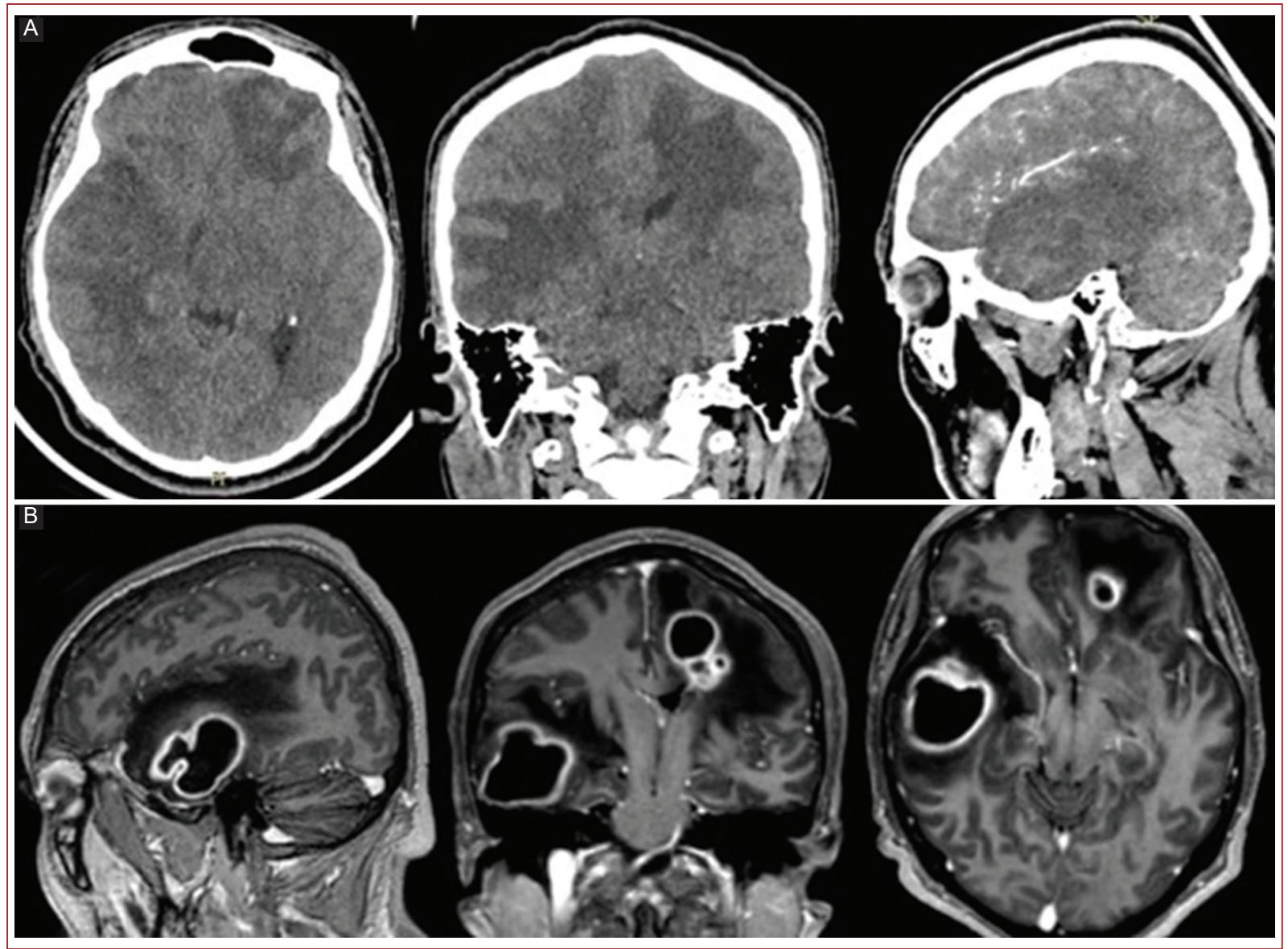

Figure 2. A: Initial single-phase skull tomography in the axial, coronal, and sagittal sections. The presence of a digitiform edema is shown in the right temporal and left frontal regions. B: Pre-operative MRI of the skull contrasted in the T1 sagittal, coronal, and axial sections. We observed a right temporal lesion with enhancement to the contrast medium, which measured $3.04 \times 3.23 \times 3.68 \mathrm{~cm}$ with an approximate volume of $18 \mathrm{ml}$, and in the left frontal region with the same characteristics but with a smaller size, $1.9 \times 1.8 \mathrm{~cm}$.

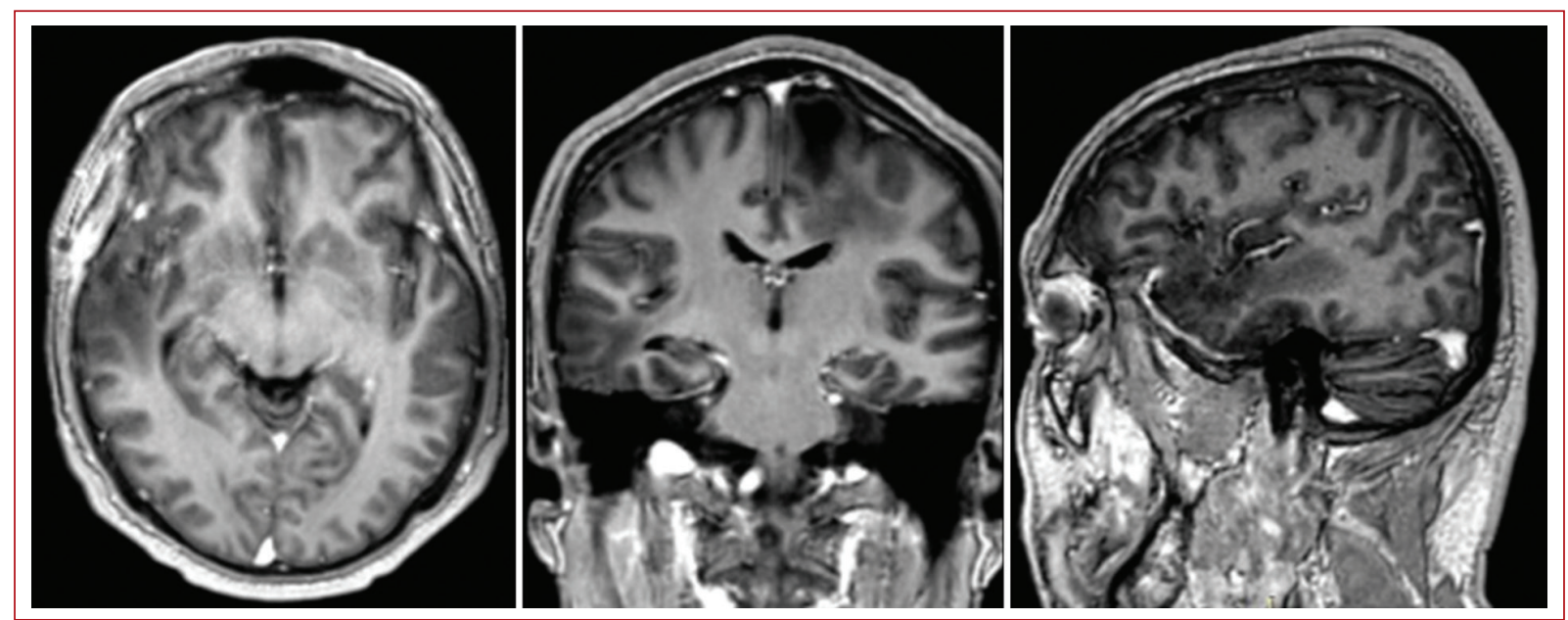

Figure 3. Post-operative T1-contrasted MRI of the skull, showing an absence of capsules and no residual injury evidence. 
formation routes are by contiguity in half the cases, and in the rest, its origin is unclear ${ }^{8}$.

The main objective in the surgical management of patients with brain abscesses is to eliminate the rise of intracranial pressure and eliminate pathogenic microorganisms ${ }^{9}$. Hence, early diagnosis, timely drainage of brain pus collection, elimination of the primary source, and management with high intravenous doses of antibiotics are fundamental for successful results in our patients ${ }^{10}$.

Evidence suggests that patients with a brain abscess larger than $2.5 \mathrm{~cm}$ in diameter ought to be treated surgically, given that results with conservative management with antibiotics have been poor ${ }^{11}$.

Classically, physicians opted for open management through craniotomy and resection of brain abscesses since this procedure had proven to be the one with the lowest recurrence and hospital stay rates ${ }^{12}$. However, in an attempt to reduce damage to healthy tissue commonly observed in these approaches, new minimally invasive techniques have been designed, such as the single-trephine neuronavigation-guided puncture and aspiration. Meng et al. compared results of patients managed through the classic approach with craniotomy and resection of the brain abscess to the single trephine aspiration and neuronavigation technique. Their findings showed that the latter approach offers less blood loss, less operating times, and a smaller incision without significant differences regarding hospital stay, mortality rates, reoperation rates, and post-operative fever ${ }^{10}$. However, routine management through craniotomy is still in the therapeutic algorithm when the abscess creates a considerable mass effect with herniation, if there is a diagnostic doubt, if the abscess is the result of a traumatic injury with foreign bodies or bone fragments, if the injury is in the posterior fossa, or if there is a recurrence after performing a minimally invasive management ${ }^{10}$. Despite this, over time, the use of craniotomy and resection for these patients' management has lowered considerably ${ }^{13}$.

\section{Conclusion}

We present the case of a patient with metastatic brain abscess secondary to multiple hepatic abscesses successfully treated through neuronavigation with the single-trephine neuronavigation-guided puncture and aspiration technique. These represent a safe and effective alternative and could avoid a large craniotomy by locating the lesion's exact location with great precision and accessing it through a minimally invasive approach, with less damage to surrounding healthy tissue, reducing possible complications and increasing the probability of therapeutic success.

\section{Ethical disclosures}

Protection of people and animals. The authors declare that no experiments were performed on humans or animals for this research.

Data confidentiality. The authors declare that they have followed their work center's protocols on the publication of patient data.

Privacy and informed consent. The authors have obtained the patients' informed consent and/or subjects referred to in the article. This document is in possession of the corresponding author.

\section{Acknowledgments}

To all medical personnel involved in the integral management of the patient.

\section{Funding}

No financing was obtained to conduct this article.

\section{Conflicts of interest}

The authors declare that there are no conflicts of interest.

\section{References}

1. Tunkel AR. Brain abscess. In: Mandell GL, Bennett JE, Dolin R, editors. Principles and Practice of Infectious Diseases. $7^{\text {th }}$ ed. Philadelphia, PA: Churchill Livingstone; 2010. p. 1265-78.

2. Mathisen GE, Johnson JP. Brain abscess. Clin Infect Dis. 1997;25:763-79.

3. Kastenbauer S, Pfister HW, Wispelwey B, Scheld WM. Brain abscess. In: Infections of the Central Nervous System. $3^{\text {rd }}$ ed. Philadelphia, PA: Lippincott Williams \& Wilkins; 2004. p. 479-507.

4. Goldman JM, Kowalec JK. Hepatic abscess and osteomyelitis from Klebsiella pneumoniae. JAMA. 1978;240:2660.

5. Chang FY, Chou MY, Fan RL, Shaio MF. A clinical study of Klebsiella liver abscess. Taiwan Yi Xue Hui Za Zhi. 1988;87:282-7.

6. Chen SC, Yen $\mathrm{CH}$, Tsao SM, Huang CC, Chen CC, Lee MC et al. Comparison of pyogenic liver abscesses of biliary and cryptogenic origin. An eight-year analysis in a University Hospital. Swiss Med Wkly. 2005;135:344-51.

7. Cheng DL, Liu YC, Yen MY, Liu CY, Shi FW, Wang LS. Pyogenic liver abscess: clinical manifestations and value of percutaneous catheter drainage treatment. J Formos Med Assoc. 1990;89:571-6.

8. DiFiglia SE, Cramer C. Friedlander's bacillus meningitis in a case with liver abscess and recurrent bacteremia and analysis of cases receiving specific therapy. N Y State J Med. 1951;51:761-5.

9. Cavus,oglu H, Kaya RA, Turkmenoglu ON, Colak I, Aydin Y. Brain abscess: analysis of results in a series of 51 patients with a combined surgical and medical approach during an 11-year period. Neurosurg Focus. 2008;24:E9.

10. Meng XH, Feng SY, Chen XL, Li C, Zhang J, Zhou T, et al. Minimally invasive image-guided keyhole aspiration of cerebral abscesses. Int $\mathrm{J}$ Clin Exp Med. 2015;8:155-63.

11. Heineman HS, Braude AI, Osterholm JL. Intracranial suppurative disease. Early presumptive diagnosis and successful treatment without surgery. JAMA. 1971;218:1542-7.

12. Sarmast AH, Showkat HI, Kirmani AR, Bhat AR, Patloo AM, Ahmad SR, et al. Aspiration versus excision: a single center experience of forty-seven patients with brain abscess over 10 years. Neurol Med Chir (Tokyo). 2012;52:724-30

13. Aras $Y$, Sabanci PA, Izgi N, Boyali O, Ozturk O, Aydoseli A, et al. Surgery for pyogenic brain abscess over 30 years: evaluation of the roles of aspiration and craniotomy. Turk Neurosurg. 2016;26:39-47. 